\title{
Plasma-induced alignment of carbon nanotubes
}

\author{
Chris Bower ${ }^{\mathrm{a})}$ \\ Department of Physics and Astronomy, University of North Carolina, Chapel Hill, North Carolina 27599 \\ Wei Zhu ${ }^{\text {b) }}$ and Sungho Jin \\ Bell Laboratories, Lucent Technologies, Murray Hill, New Jersey 07974 \\ Otto Zhou \\ Department of Physics and Astronomy and Curriculum in Applied and Materials Science, \\ University of North Carolina, Chapel Hill, North Carolina 27599
}

(Received 6 April 2000; accepted for publication 13 June 2000)

\begin{abstract}
Uniform films of well-aligned carbon nanotubes have been grown using microwave plasma-enhanced chemical vapor deposition. It is shown that nanotubes can be grown on contoured surfaces and aligned in a direction always perpendicular to the local substrate surface. The alignment is primarily induced by the electrical self-bias field imposed on the substrate surface from the plasma environment. It is found that switching the plasma source off effectively turns the alignment mechanism off, leading to a smooth transition between the plasma-grown straight nanotubes and the thermally grown "curly" nanotubes. The nanotubes grow at a surprisingly high rate of $\sim 100 \mathrm{~nm} / \mathrm{s}$ in our plasma process, which may be important for large-scale commercial production of nanotubes. (C) 2000 American Institute of Physics. [S0003-6951(00)02532-8]
\end{abstract}

Carbon nanotubes are graphitic tubules with nanometersize diameters and very large aspect ratios. ${ }^{1}$ They exhibit highly anisotropic physical and chemical properties that are attractive for applications in nanoscale devices and technologies. ${ }^{2-4}$ To realize their potentials, controlled growth of well-aligned nanotubes is essential. Aligned carbon nanotubes have been previously obtained using thermal chemical vapor deposition (CVD) by growing nanotubes on flat substrates of porous silicon ${ }^{5,6}$ and within membranes containing highly ordered pores. ${ }^{7}$ The alignment mechanism was explained to be due to the constraint placed on the nanotube growth direction by the pores in the substrate as well as the Van der Waals interaction between neighboring nanotubes. Ren et al. ${ }^{8}$ found that large (>100 $\mathrm{nm}$ diameter) nanotubes could be grown in a highly aligned manner using hot filament direct-current (dc) plasma enhanced chemical vapor deposition. The nanotubes grown in Ren's study had catalyst particles at the nanotube tips, and the growth and alignment mechanisms are not clear.

Here we report the growth of aligned carbon nanotubes using high-frequency, microwave plasma enhanced chemical vapor deposition (MPECVD). The nanotube growth was found to always occur perpendicular to the local substrate surface regardless of the substrate tilt or shape, with an extreme-case example of conformal radial growth of nanotubes on the surface of a hair-thin optical fiber glass. The ability to quickly and easily grow films of aligned nanotubes on contoured surfaces will facilitate the use of nanotubes for many technological applications. We show that the alignment is not a result of Van der Waals interactions between neighboring nanotubes, but is primarily induced by the electrical self-bias imposed on the substrate surface from the microwave plasma. We also show that turning off the plasma

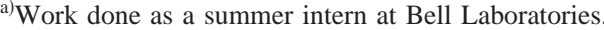

b) Author to whom correspondence should be addressed; electronic mail: wzhu@bell-labs.com
}

effectively turns off the alignment mechanism and demonstrates the capability of intentionally introducing a smooth junction between the plasma-grown straight nanotube and the thermally grown curly nanotube.

The microwave plasma enhanced CVD system that was used to grow the multiwall carbon nanotubes consists of a $2.45 \mathrm{GHz}, 5 \mathrm{~kW}$ microwave power supply with a rectangular waveguide that is coupled to a 6-in.-inner-diameter stainlesssteel cylindrical growth chamber and a molybdenum substrate stage with a radio-frequency (rf) graphite heater that allows control of the substrate temperature independent of the plasma power. A thin film of catalyst metal (cobalt) was sputter deposited on the substrate surface prior to the CVD growth. The sample was then transferred in air to the growth chamber and heated to $825^{\circ} \mathrm{C}$ in flowing hydrogen at a pressure of 20 Torr. A $1 \mathrm{~kW}$ microwave plasma was then turned on, and the hydrogen environment was completely replaced with a mixture of ammonia and acetylene to start the nanotube growth. Typically, a growing nanotube film can be visibly seen through a chamber window on the substrate about $10 \mathrm{~s}$ after the introduction of acetylene. Total gas flow rates of acetylene $\left(\mathrm{C}_{2} \mathrm{H}_{2}\right)$ and ammonia $\left(\mathrm{NH}_{3}\right)$ were controlled at $200 \mathrm{sccm}$, and the mass flow ratio of $\mathrm{C}_{2} \mathrm{H}_{2}$ over $\mathrm{NH}_{3}$ was varied in the range of 10\%-30\%. Electron microscopy and $\mathrm{x}$-ray diffraction were employed to characterize these nanotube films.

The alignment of carbon nanotubes was first demonstrated on flat Si substrates placed in either a vertical or tilted position in addition to the normal horizontal position on the substrate stage [see Fig. 1(a)]. We found that the nanotubes always grow perpendicular to the substrate surfaces regardless of the substrate position. Figure 1(b) shows a representative scanning electron microscopy (SEM) micrograph of such aligned nanotubes grown on a cobalt-coated $(\sim 2 \mathrm{~nm}$ thick) silicon substrate. They are multiwalled nanotubes about $30 \mathrm{~nm}$ in diameter and $12 \mu \mathrm{m}$ long. The inset in Fig. 1(b) shows the cobalt islands which formed during the tem- 
(a)

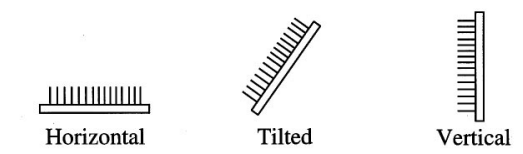

(b)

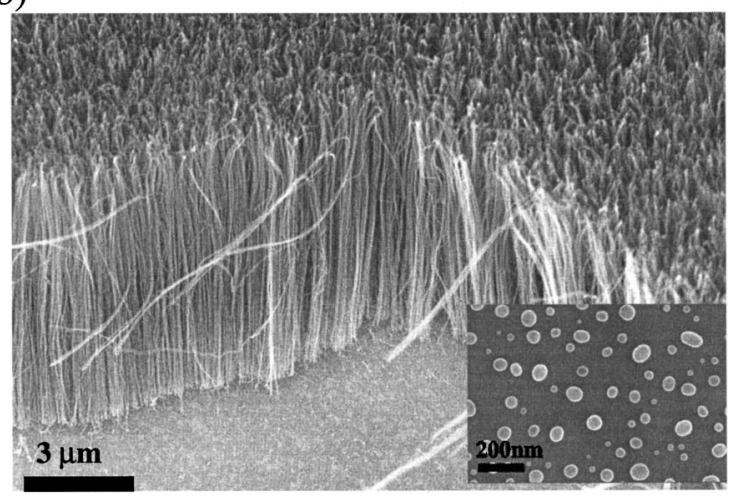

(c)

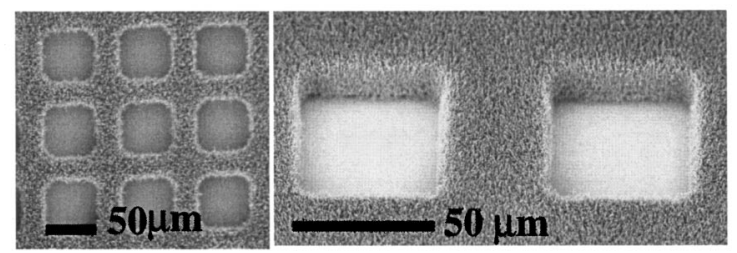

FIG. 1. (a) Schematics showing the perpendicular alignment of nanotube growth regardless of the substrate position. (b) An SEM image that shows the growth of well aligned nanotubes. The inset shows the cobalt islands that formed prior to the nanotube growth. (c) SEM images showing patterned nanotube growth as a result of patterning the cobalt catalyst prior to the growth.

perature ramp-up before the introduction of acetylene, and which served as the nanotube nucleation sites and dictated the density of nanotubes. The nanotube-to-nanotube distance corresponds to the spacing between the cobalt islands, which was measured to be about $150 \mathrm{~nm}$ on average. This corresponds to a density of $4.4 \times 10^{9}$ nanotubes $/ \mathrm{cm}^{2}$. We note that the spacing of our catalyst islands is larger then that reported for other growth methods. ${ }^{6,8}$ The growth rate was surprisingly high $(\sim 100 \mathrm{~nm} / \mathrm{s})$, with the entire growth of the $12 \mu \mathrm{m}$ length taking only $2 \mathrm{~min}$. Such a high growth speed for nanotubes may be important for successful commercialization of nanotube applications. No cobalt particles were observed at the nanotube tips with SEM. X-ray diffraction studies with an area detector, similar to those performed by Jin et al., ${ }^{9}$ showed that the nanotubes were aligned to within 8 deg of the surface normal. The cobalt catalyst could be patterned by photolithography or shadow mask, allowing the growth of nanotubes in selected areas. As shown in Fig. 1(c), the aligned nanotubes only grow in the areas where cobalt was present, with no nanotubes seen on the bare Si surface.

This remarkable tube alignment was obtainable only from the plasma environment, because a thermal process alone under otherwise identical conditions yielded randomly oriented curly nanotubes of similar diameter. This suggests that the nanotube orientation is influenced by the self-bias potential established on the immersed substrate surface in the high frequency plasma, the field line of which is invariably terminated perpendicular to the surface. ${ }^{10}$ The electrostatic force would force these one-dimensional tubular structures to Downloaded 26 Nov 2002 to 152.26 .144 . Redistribution subject (a)

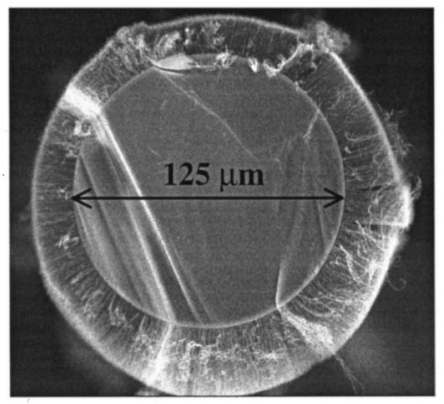

(b)

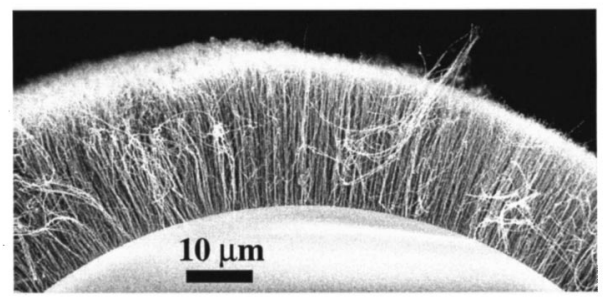

(c) (d)

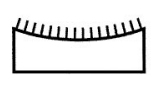

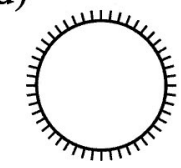

(e)

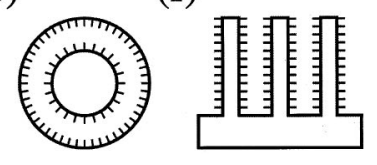

FIG. 2. (a) An SEM micrograph showing the radially grown nanotubes on the surface of a $125-\mu \mathrm{m}$-diam optical fiber. (b) A close-up micrograph showing the conformally perpendicular nature of the nanotube growth on the fiber. (c)-(f) are examples of nonplanar, complex surfaces where nanotubes can be conformally grown perpendicular to the local surface.

align with the field direction, an energetically most favorable orientation. This is similar to the electrostatic deflections of nanotubes demonstrated by Poncharal et al. ${ }^{11} \mathrm{We}$ estimate that, for a cold microwave plasma of ammonia and acetylene mixture at $1 \mathrm{~kW}$ input power and 20 Torr pressure, the selfbias potential is on the order of $10 \mathrm{~V}$. This potential is estimated to drop across a plasma sheath of about $100 \mu \mathrm{m}$, generating an electric field perpendicular to the substrate with a magnitude of $0.1 \mathrm{~V} / \mu \mathrm{m}$ in the vicinity of the surface. Furthermore, since the self-bias potential is proportional to the mass of the ions $\left[V=\left(k T_{e} / 2 e\right) \ln \left(m_{i} / 2.3 m_{e}\right)\right],{ }^{10}$ where $k$ is Boltzmann's constant, $T_{e}$ is the electron temperature, $e$ is the charge of the electron, and $m_{i}$ and $m_{e}$ are the mass of ions and electrons, respectively, the use of relatively heavier ammonia plasma is helpful in establishing a stronger local field at the surface, compared with other types of much lighter-mass plasmas such as a hydrogen plasma. This could explain why others have grown randomly oriented nanotubes in a similar MPECVD system using hydrogen based plasma. ${ }^{12,13} \mathrm{~A}$ recent letter by Murakami et al. ${ }^{14}$ did show aligned nanotube growth using a hydrogen-based plasma, but the degree of alignment was not reported.

Exploiting the perpendicular nature of the self-induced field near the surface, we then successfully grew aligned nanotubes on a variety of contoured surfaces. A striking example, as shown in Figs. 2(a) and 2(b), is that aligned nanotubes can be grown on the circumferential surface of a hairthin, telecom-grade $\mathrm{SiO}_{2}$ optical fiber with about $125 \mu \mathrm{m}$ in diameter. Each nanotube is seen pointing radially outward o AIP license or copyright, see http://ojps.aip.org/aplo/aplcr.jsp 
(a)

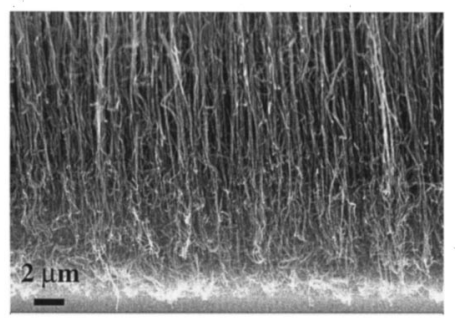

(b)

(c)

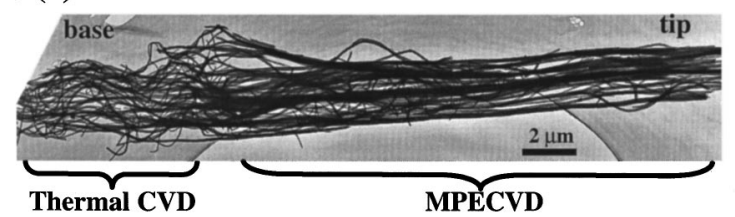

FIG. 3. (a) An SEM micrograph and (b) a schematic showing the straight/ curled nanotube structure produced by an alternating plasma and thermal process (a 2 min plasma process followed by a $70 \mathrm{~min}$ thermal process), indicating both the field induced alignment effect and the base growth mechanism. (c) is a TEM micrograph showing a bundle of nanotubes with the upper portion straight and the lower portion curled

and perpendicular to the surface. This is another demonstration of the role of the local electric field played in the nanotube alignment. We can envision a number of interesting and potentially useful structures, in which aligned nanotubes are grown on geometrically different types of surfaces, as schematically illustrated in Figs. 2(c)-2(f). These configurations include surfaces of a concaved electrode, sphere (resembling a sea urchin), concentric hollow cylinder (both inner and outer surfaces), and parallel plates, each having important advantages in a number of devices. For example, aligned nanotubes grown on a concave-shaped cathode surface will have electron beam self-focusing effects during field emission. Nanotubes grown on the outer surface of a sphere, round rod, or cylinder can be used more effectively as a radially emitting electron source for possibly generating radiative $\mathrm{x}$ rays for tumor treatments. Nanotubes grown on parallel plates, concentric cylinders, or a rod-cylinder combination may be utilized as high-surface-area electrochemical electrodes, hydrogen storage media, or catalysts.

In an attempt to further elucidate the alignment mechanisms, the plasma was turned off after a few minutes of growth and the nanotubes were allowed to continue growing in a thermal CVD environment. The cross-sectional structure of the resulting nanotube film is shown by the SEM micrographs in Fig. 3(a) and illustrated schematically in Fig. 3(b). During the first two minutes of growth, the plasma was on, and the nanotubes grew in an aligned manner. During the subsequent 70 min the plasma was off, and the nanotubes were seen to grow in a random or curly fashion. This type of straight to curly transitional structure is again shown in Fig. 3(c), in which a transmission electron microscopy (TEM) micrograph shows a bundle of nanotubes with their upper section straight and lower section curled. The nanotube growth rate is more than 40 -fold slower $(\sim 150 \mathrm{~nm} / \mathrm{min})$ without the plasma enhancement, possibly due to the different types or quantities of reactive species produced in a thermal process. It is clear that the new curly growth took place at the base, supporting the base-growth mechanism in our processes. It also clearly indicates that the alignment is asso-

Downloaded 26 Nov 2002 to 152.2.144. Redistribution subject ciated with the plasma environment. If the alignment was due to Van der Waals forces or steric hindrance between closely packed nanotubes, the growth would have continued in an aligned manner after the plasma was turned off.

The ability to control the nanotube alignment mechanism could be of profound importance for building useful device structures with nanotubes. Here, we have shown that by turning on or off the plasma source, which essentially switches on or off the alignment (i.e., linear growth) mechanism, we can controllably grow either straight or curly nanotubes in a repeated manner. One can envision that finer and optimized control of the growth environment could lead to the ability to controllably introduce a desired junction-type defect at the physical junction of a straight and curled nanotube at any selected location along the length of the nanotube by pulsing the plasma on or off. The introduction of these junction-type defects often accompanies changes in electrical properties such as in electrical resistivity and band gap. ${ }^{3}$ As a result, they could function as important device nodes such as metal-semiconductor intramolecular junctions. ${ }^{15}$ The ability to deliberately introduce junction-type defects along the length of otherwise defect-free nanotubes provides a practical means to grow such carefully designed structures for potential active molecular-level devices.

In summary, we have demonstrated the growth of aligned carbon nanotubes using microwave plasma enhanced chemical vapor deposition (MPECVD). The nanotubes are grown always perpendicular to the local substrate surface regardless of the surface tilt or shape. The electrical self-bias imposed on the substrate surface appears to be the primary mechanism responsible for the conformal alignment. We show that by turning off the plasma source, which effectively turns off the alignment mechanism, a smooth transition can be introduced at the physical junction between a plasmagrown straight nanotube and a thermally grown curly nanotube.

The authors thank the technical help from G. Kammlott (SEM), H. Krautter (XPS), and K. Evans-Lutterodt (x-ray diffraction). The authors also wish to acknowledge D. J. Werder, R. Tung, and A. G. Ramirez for useful discussions.

${ }^{1}$ S. Iijima, Nature (London) 354, 56 (1991).

${ }^{2}$ B. I. Yakobson and R. E. Smalley, Am. Sci. 85, 324 (1997).

${ }^{3}$ M. S. Dresselhaus, G. Dresselhaus, and P. C. Eklund, Science of Fullerences and Carbon Nanotubes (Academic, San Diego, CA, 1996).

${ }^{4}$ C. Dekker, Phys. Today 52, 22 (1999).

${ }^{5}$ W. Z. Li, S. S. Xie, L. X. Qian, B. H. Chang, B. S. Zou, W. Y. Zhou, R. A. Zhao, and G. Wang, Science 274, 1701 (1996).

${ }^{6}$ S. Fan, M. G. Chapline, N. R. Franklin, T. W. Tombler, A. M. Cassell, and H. Dai, Science 283, 512 (1999).

${ }^{7}$ J. Li, C. Papadopoulos, and J. M. Xu, Appl. Phys. Lett. 75, 367 (1999).

${ }^{8}$ Z. F. Ren, Z. P. Huang, J. W. Xu, J. H. Wang, P. Bush, M. P. Siegal, and P. N. Provencio, Appl. Phys. Lett. 282, 1105 (1998).

${ }^{9}$ L. Jin, C. Bower, and O. Zhou, Appl. Phys. Lett. 73, 1197 (1998).

${ }^{10}$ B. Chapman, Glow Discharge Processes (Wiley, New York, 1980).

${ }^{11}$ P. Poncharal, Z. L. Wang, Z. Ugarte, and W. A. de Heer, Science 283, 1513 (1999).

${ }^{12}$ L. C. Qin, D. Zhou, A. R. Krauss, and D. M. Gruen, Appl. Phys. Lett. 72, 3437 (1998)

${ }^{13}$ O. M. Kuttel, O. Groening, C. Emmenegger, and L. Schlapbach, Appl. Phys. Lett. 73, 2113 (1998)

${ }^{14}$ H. Murakami, M. Hirakawa, C. Tanaka, and H. Yamakawa, Appl. Phys. Lett. 76, 1776 (2000).

${ }^{15}$ Z. Yao, H. W. Ch. Postma, L. Balents, and C. Dekker, Nature (London) 402, 273 (1999).

AIP license or copyright, see http://ojps.aip.org/aplo/aplcr.jsp 